\title{
A Study on Alexithymia, Quality of Life, and Facial Emotion Recognition Abilities in Somatoform Disorders
}

\author{
${ }^{1}$ Vinuprasad Venugopalan, ${ }^{2}$ Manas Elkal, ${ }^{3}$ Rishikesh V Behere, ${ }^{4}$ Samir K Praharaj, ${ }^{5}$ Haridas Kanaradi
}

\begin{abstract}
Aim: To assess alexithymia and quality of life among patients of somatoform disorders (SFD) compared with healthy control subjects and to assess the association between alexithymia and facial emotion recognition ability and its influence on quality of life within diagnostic subgroups of SFD.
\end{abstract}

Materials and methods: Forty-three patients diagnosed to have SFD (International Classification of Diseases (ICD)-10) were assessed on the World Health Organization (WHO) SFD symptom checklist, Toronto Alexithymia Scale-26 (TAS-26), Tool for Recognition of Emotions in Neurological Disorders (TRENDS) and WHO Quality of Life (QOL) BREF to measure quality of life. They were compared with a control group of 47 healthy subjects.

Results: Patients with SFD had greater alexithymia scores and poorer quality of life compared with controls. A novel observation was the inverse correlation between alexithymia and facial emotion recognition deficit, specifically in the diagnostic subgroup of persistent somatoform pain disorder compared with other diagnostic subtypes.

Conclusion: Alexithymia is an important trait influencing quality of life, especially in patients with a diagnosis of persistent somatoform pain disorder and is associated with deficits in facial emotion recognition.

Clinical significance: Association between alexithymia and facial emotion recognition is predominant in patients with somatoform pain disorder. Psychological interventions focusing on improving social cognition could potentially play a role in improving the quality of life in patients with persistent somatoform pain disorder.

Keywords: Alexithymia, Case-control study, Facial emotion recognition, Quality of life, Somatoform disorder.

${ }^{1}$ Assistant Professor, ${ }^{2}$ Psychiatrist, ${ }^{3}$ Fellow and Associate Consultant, ${ }^{4}$ Professor, ${ }^{5}$ Associate Professor (Rtd)

${ }^{1}$ Department of Psychiatry, P K Das Institute of Medical Sciences, Ottapalam, Kerala, India

${ }^{2}$ District Mental Health Project, Udupi, Karnataka, India

${ }^{3}$ Psychiatry Unit, KEM Hospital Research Center, Pune, Maharashtra, India

${ }^{4,5}$ Department of Psychiatry, Kasturba Medical College, Manipal Karnataka, India, India

Corresponding Author: Vinuprasad Venugopalan, Assistant Professor, Department of Psychiatry, P K Das Institute of Medical Sciences, Ottapalam, Kerala, India, e-mail: vinuprasadvgm 2000@yahoo.com
How to cite this article: Venugopalan V, Elkal M, Behere RV, Praharaj SK, Kanaradi H. A Study on Alexithymia, Quality of Life, and Facial Emotion Recognition Abilities in Somatoform Disorders. J Postgrad Med Edu Res 2018;52(3):110-116.

Source of support: Nil

Conflict of interest: None

\section{INTRODUCTION}

Somatoform disorders refer to a group of psychiatric disorders characterized by often multiple and variable somatic symptoms that are commonly seen in general medical practice and primary care but that defy medical explanation. ${ }^{1}$ Symptoms of large proportions of patients remain unexplained even after comprehensive medical assessment. $^{2}$ As patients realize that their symptoms are physical rather than mental, they are more likely to visit a physician rather than a psychiatrist. ${ }^{3}$ Somatoform disorders occupy a considerable proportion of patient load in outpatient units and inpatient care facilities in both Eastern and Western communities. It has been estimated that somatization disorder forms $3 \%$ and hypochondriasis forms $1 \%$ of patient load in primary care clinics across 14 countries. ${ }^{4}$ Though the extent of the problem is significant, the importance given by the scientific community is much less taking into account its "contribution" in the utilization of healthcare facilities.

Somatoform disorders first entered modern-day classificatory system in Diagnostic and Statistical Manual (DSM)-III. These disorders are currently classified as somatic symptom disorder in the DSM version 5. In ICD-10, it includes diagnoses like somatization disorder, undifferentiated SFD, hypochondriacal disorder, somatoform autonomic dysfunction, persistent somatoform pain disorder, and SFD unspecified.

Steckel et $\mathrm{al}^{5}$ defined somatization in 1943 as the process of a "bodily disorder" occurring as the expression of a "deep-seated neurosis." However, as argued by Kellner, "empirical studies suggest that there is no single theory that can adequately explain somatization, which is not only multifactorially determined but is an exceedingly complex phenomenon." Furthermore, treatment strategies derived from somatization theories have not proven effective.

Attempts to explain the attributing and causative factors associated with the disorder have been varied. 
Somatosensory amplification is considered as the major contributing factor of somatization. But somatosensory amplification is neither sensitive nor specific to SFDs. Many factors like anxiety, depression, neuroticism, and alexithymia may also contribute. ${ }^{7}$

\section{Alexithymia}

Alexithymia is a concept characterized by inability to describe and identify one's own feelings, the absence of fantasies, and the utilization of an externally oriented analytical cognitive style. ${ }^{8}$ The core characteristics of alexithymia are marked dysfunction in emotional awareness, social attachment, and manner of interpersonal relating. ${ }^{9}$ Alexithymia is prevalent in approximately $10 \%$ of the general population and is known to be comorbid with a number of psychiatric conditions. The same phenomenon is observed in various medical conditions like peptic ulcer, inflammatory bowel disease, hypertension, and chronic pain. ${ }^{10}$

Alexithymic features were first assumed to be typical of patients with classical psychosomatic diseases. Various studies have consistently demonstrated alexithymia in SFD. ${ }^{11-15}$ Cox et al ${ }^{13}$ found prevalence of alexithymia to be $53 \%$ among patients with unexplained chronic pain. Bach and Bach ${ }^{12}$ found alexithymia to be significantly high in patients with SFD compared with medically ill. However, further studies have shown that an alexithymic communicative style is not specific to patients with classical psychosomatic diseases. In a study by Duddu et $\mathrm{al}^{7}$ comparing alexithymia scores among a group of patients of depression and SFD and normal controls, they found that alexithymia and difficulty in expressing feelings were associated with psychological attribution of innocuous bodily sensations in the SFD group, suggesting that alexithymic subjects are more likely to psychologize bodily symptoms than non-alexithymic subjects. They also found that while total alexithymia scores did not differentiate somatoform from depressive disorders, the two diagnostic groups did differ insofar as subjects with depression demonstrated greater difficulty in expressing feelings. The presence of alexithymia can lead to a poor quality of life in these patients. Garcia Nuñez et $\mathrm{al}^{16}$ found that alexithymia plays a major role in the reduction of quality of life in patients with SFD. Subric-Wrana et $\mathrm{al}^{17}$ found deficits in emotional awareness and theory of mind functioning and emotional awareness in patients with SFD.

Recent progress in neuroimaging studies on alexithymia has provided important information on the neural basis of alexithymia. In a study examining brain responses underlying affect dysregulation, a significant positive relation between the size of the right anterior cingulate cortex and alexithymia as measured with the TAS in healthy subjects was found. ${ }^{18}$
Facial emotion recognition is a very important component of social cognition. Neuroanatomical substrates involved in facial emotion recognition like amygdala, insula, and anterior cingulate gyrus and orbitofrontal cortex are also found to play a part in alexithymia. ${ }^{19}$

Pedrosa Gil et $\mathrm{al}^{20}$ demonstrated a deficit in facial emotional recognition in 20 patients with SFD which was attributable to concurrent alexithymia. They also commented that neither depression nor anxiety was significantly related to emotion recognition accuracy, suggesting that these variables did not contribute to the emotion recognition deficit. Schönenberg et $\mathrm{al}^{21}$ studied 19 female patients with persistent SFD for alexithymia and facial emotion recognition deficits. They found impaired mentalizing skills and increased alexithymic traits in these patients in comparison with healthy controls. However, they found no difference in facial recognition abilities between the two groups.

However, our understanding of alexithymia and its relation to facial emotion recognition abilities, quality of life, in subgroups of SFD is not well understood. In this study, we aim to examine (1) alexithymia, facial emotion recognition ability and quality of life in patients with SFD as compared with healthy control subjects and (2) the association between alexithymia, facial emotion recognition ability, and quality of life between diagnostic subgroups of SFD.

\section{MATERIALS AND METHODS}

\section{Subjects}

The study was conducted at the Psychiatry Department of Kasturba Medical College, Manipal, a tertiary care center in coastal Karnataka, India. Subjects in age range of 18 to 55 years of either sex who were able to read and write Kannada were recruited after obtaining informed consent from patients attending psychiatric outpatient services of our hospital and received a diagnosis from the somatoform group of disorders as per ICD-10 criteria. The diagnosis was confirmed independently by two psychiatrists. Patients with comorbid psychotic, depressive, anxiety disorder, or substance use disorder (ICD-10) were excluded. Comorbid diagnosis of dysthymia was not considered as exclusion and such patients were included in the study. Most of these patients were naïve to psychotropic medications. The study was approved by the institutional ethics committee, Kasturba Hospital, Manipal.

The control group was selected by word of mouth from hospital employees and their relatives. They were screened for any symptoms of psychological distress on the general health questionnaire $5 .^{22}$ Only those subjects with a score $\leq 1$ were recruited. Control subjects with a past history of any psychiatric disorder were excluded from the study. 


\section{Tools}

Sociodemographic and clinical details were recorded using a pro forma designed for the study. Toronto alexithymia scale, a 26-item self-report questionnaire was used to study alexithymia. It was developed by Taylor et $\mathrm{al}^{23}$ using a factor analytic, construct-oriented approach. Validated Kannada version of the TAS reported by Sriram et $\mathrm{a}^{10}$ was used in this study to quantify alexithymia. Each question is answered according to Likert scale from 1 to 5 . The scale is shown to have high internal consistency. ${ }^{24}$ Factor analysis studies give four factors in alexithymia. They are: (TAS-1) difficulty in identifying feelings and bodily sensations, (TAS-2) externally oriented thinking, (TAS-3) difficulty expressing feelings, and (TAS-4) Inability to interpret bodily manifestations of emotions. ${ }^{11}$ To evaluate somatoform symptoms, the WHO SFD symptom checklist ${ }^{25}$ was used. The checklist lists 60 symptoms in the domains of pain, cardiovascular, autonomic, gastrointestinal, and genitourinary. Patients have to indicate the presence or absence of each symptom and the total number of symptoms experienced was calculated. The WHO QOL-BREF ${ }^{26}$ was used to study the quality of life. This is an abbreviated version of WHO QOL 100 introduced by the WHO in 1996. It is a 24-item self-rated questionnaire which gives information on four major domains of quality of life like physical (QOL-1), psychological (QOL-2), social relationship (QOL-3), and environmental (QOL-4). The hospital anxiety depression scale (HADS) ${ }^{27}$ was used to measure the extent of anxiety/depressive symptoms in both patients and controls. A score of greater than 11 on the scale indicates significant anxiety and depressive symptoms. Tool for recognition of emotions in neuropsychiatric disorders $(\text { TRENDS })^{28}$ was used to study facial emotion recognition. The TRENDS is a tool validated for use in the Indian population, which captures the full range and nature of emotional expressions akin to real-life situations. It consists of two arms-the static (still photographs) and the dynamic (videos) arm. Fifty-two still images of four actors (one young male, one young female, one old male, and old female) emoting six basic emotions (happy, sad, fear, anger, surprise, and disgust) at two intensities with neutral and 28 videos were used.

\section{Statistical Analysis}

Statistical Package for the Social Sciences version 16.0 was used for the statistical analysis. The scores on TAS-26 and QOL-BREF were computed on the domains as described earlier. The variables were normally distributed as assessed on the Kolmogorov-Smirnov test. The demographic variables and scores on HADS were compared on independent samples $t$ test or chi-square test as applicable. The TRENDS accuracy score (TRACS), which is the total number of images correctly identified (out of maximum of 80), was computed. The scores on TAS-26, QOL domains, and TRACS were compared between and patients and control groups and across diagnostic subtypes by analysis of covariance (ANCOVA) with scores on the HADS as covariate. Significance was set at $p<0.005$ after applying Bonferroni correction for multiple comparisons. Pearson's correlation analysis was performed to look for any correlation between the variables.

\section{RESULTS}

Sample size was 43 in the patient group and 47 in the control group. Among the patients, 17 (39.5\%) had a diagnosis of persistent somatoform pain disorder, and 26 $(60.5 \%)$ patients had a diagnosis of undifferentiated SFD or somatoform autonomic dysfunction. Patient and control groups were comparable on sociodemographic parameters like age, gender, and years of education (Table 1).

Twenty-seven out of 43 patients had alexithymia $(62.8 \%)$ and scored above cut-off of 72 on the TAS-26. On the HADS, patients scored significantly higher than controls ( $p<0.001)$; 15 patients scored greater than 11 on the HADS, indicating significant depressive or anxiety

Table 1: Comparison of demographic and clinical characteristics between patients and controls

\begin{tabular}{|c|c|c|c|c|c|}
\hline \multirow{2}{*}{ Age, mean (SD) } & & \multirow{2}{*}{$\begin{array}{l}\begin{array}{l}\text { Patients } \\
(n=43)\end{array} \\
40(8.7)\end{array}$} & $\begin{array}{l}\text { Controls } \\
(n=47)\end{array}$ & $t / \chi^{2}$ & $p$-value \\
\hline & & & $38.7(8.8)$ & 0.9 & 0.3 \\
\hline Education years, mean (SD & & $8.8(4.1)$ & $10.0(3.9)$ & 1.5 & 0.1 \\
\hline HADS Score, mean (SD) & & $10.6(7.3)$ & $3.0(3.7)$ & $6.2^{*}$ & $<0.001$ \\
\hline \multicolumn{2}{|c|}{ Duration of illness (years), mean (SD) } & $4.0(3.2)$ & & & \\
\hline \multirow[t]{2}{*}{ Gender, n (\%) } & Male & 24 & 24 & 0.2 & 0.6 \\
\hline & Female & 19 & 23 & & \\
\hline \multirow[t]{3}{*}{ Diagnosis (ICD-10), n (\%) } & Persistent somatoform pain disorder & $16(37.2)$ & - & - & - \\
\hline & Undifferentiated somatoform disorder & $20(46.5)$ & - & & \\
\hline & Somatoform autonomic dysfunction & $7(16.3)$ & - & & \\
\hline \multirow[t]{2}{*}{ Comorbid diagnosis, $\mathrm{n}(\%)$} & Dysthymia & $6(14)$ & - & - & - \\
\hline & Migraine & $2(4.7)$ & - & & \\
\hline
\end{tabular}


Alexithymia, Quality of Life, and Facial Emotion Recognition Abilities

Table 2: ANCOVA showing comparisons of alexithymia and quality of life scores between patients and controls and across diagnostic subtypes on TAS and QOL after controlling for HADS score

\begin{tabular}{|c|c|c|c|c|c|c|c|c|c|c|}
\hline & $\begin{array}{l}\text { Patients } \\
(n=39) \\
\text { mean (SD) }\end{array}$ & $\begin{array}{l}\text { Controls } \\
(n=47) \\
\text { mean (SD) }\end{array}$ & $F 1$ & p-value & $\begin{array}{l}\text { Partial eta } \\
\text { squared } \\
\text { (effect } \\
\text { size) }\end{array}$ & $\begin{array}{l}\text { Somatoform } \\
\text { disorders other } \\
\text { than pain disorder } \\
(n=23) \text { mean } \\
(S D)\end{array}$ & $\begin{array}{l}\text { Persistent } \\
\text { somatoform } \\
\text { pain disorder } \\
(n=16) \\
\text { mean }(S D)\end{array}$ & $F 2$ & $p$-value & $\begin{array}{l}\text { Partial eta } \\
\text { squared } \\
\text { (effect } \\
\text { size) }\end{array}$ \\
\hline TAS 1 & $12.17(4.4)$ & $10.9(3.6)$ & 2.5 & 0.09 & 0.06 & $12.3(4.5)$ & $13.3(4.1)$ & 0.5 & 0.6 & 0.03 \\
\hline TAS 2 & $12.4(5.2)$ & $11.1(3.1)$ & 1.2 & 0.3 & 0.03 & $12.3(4.9)$ & $12.5(5.7)$ & 0.04 & 0.9 & 0.002 \\
\hline TAS 3 & $16.9(4.7)$ & $14.9(3.6)$ & 3.1 & $0.05^{\#}$ & 0.07 & $16.2(5.0)$ & $17.9(4.3)$ & 1.1 & 0.7 & 0.02 \\
\hline TAS 4 & $15.4(4.9)$ & $11.8(4.3)$ & 6.4 & $0.003^{*}$ & 0.13 & $14.9(4.8)$ & $16.1(5.1)$ & 2.4 & 0.1 & 0.1 \\
\hline TAS Total & $75.7(13.6)$ & $63.8(9.5)$ & 12.03 & $<0.001^{*}$ & 0.23 & $72.0(11.5)$ & $80.9(15.0)$ & 0.2 & 0.8 & 0.01 \\
\hline QOL 1 & $18.5(4.2)$ & $28.04(4.3)$ & 60.9 & $<0.001^{*}$ & 0.6 & $18.6(4.1)$ & $18.3(4.5)$ & 0.1 & 0.9 & 0.01 \\
\hline QOL 2 & $16.0(4.3)$ & $25.5(2.9)$ & 72.6 & $<0.001^{*}$ & 0.64 & $17.0(4.6)$ & $14.5(3.3)$ & 2.2 & 0.1 & 0.1 \\
\hline QOL 3 & $10.4(1.9)$ & $12.8(1.7)$ & 20.4 & $<0.001^{*}$ & 0.33 & $10.5(1.8)$ & $10.2(2.1)$ & 0.4 & 0.6 & 0.02 \\
\hline QOL 4 & $25.5(4.2)$ & $33.1(4.3)$ & 33.9 & $<0.001^{*}$ & 0.45 & $25.8(3.8)$ & $25.1(4.7)$ & 0.2 & 0.8 & 0.009 \\
\hline TRACS & $44.3(9.4)$ & $63.7(9.5)$ & 49.8 & $<0.001^{*}$ & 0.5 & $47.6(5.5)$ & $39.6(11.7)$ & 4.7 & $0.02^{\#}$ & 0.2 \\
\hline
\end{tabular}

F1: ANCOVA statistic value for comparison between patient and control groups with scores on HADS as covariate; F2: ANCOVA statistic value for comparison between diagnostic subgroups with scores on HADS as covariate; ${ }^{*}$ significance at $p<0.05$; * ${ }^{\text {significance at } p<0.005}$ (after applying Bonferroni correction for multiple comparison)

symptoms, and $6(14 \%)$ patients had received a comorbid diagnosis of dysthymia. The scores on TRENDS, TAS-26, and QOL domains were compared between the patients and control groups and across diagnostic subtypes by ANCOVA with scores on the HADS as covariate to control for the possible influence of depressive and anxiety symptoms (Table 2). The HADS scores were available for 39 patients and these patients were included for the ANCOVA analysis. There was a significant main effect of group on TRACS, TAS-3, TAS-4, total TAS scores, and all four domains of quality of life $(\mathrm{p}<0.005)$ with the patient group experiencing greater emotion recognition deficits, alexithymia, and poorer quality of life as compared with healthy controls. There was a significant difference in TRAC scores between the diagnostic subgroups of persistent somatoform pain disorder and undifferentiated SFD.

On correlation analysis in the patient group $(n=43)$, TAS-1 scores correlated negatively with facial emotion recognition ability $(r=-0.3, p=0.03)$. There was a significant negative correlation between TAS-3 (difficulty expressing feelings) and QOL2 scores (psychological domain) $(r=-0.38, p=0.01)$ and between QOL3 scores (social domain) and TAS-2 scores (externally oriented thinking) $(\mathrm{r}=-0.3, \mathrm{p}=0.02)$. On examining correlations within diagnostic subtypes, only persistent somatoform pain disorder group retained the correlation between TRACS and TAS- $1(\mathrm{r}=-0.5, \mathrm{p}=0.02)$, and between QOL3 and TAS-2 $(\mathrm{r}=-0.6, \mathrm{p}=0.01)$.

\section{DISCUSSION}

In this study, we examined the association between alexithymia and quality of life in patients with SFD as compared with healthy control subjects. The main findings of the study were: (1) Patients with SFD experienced greater emotion recognition deficits, alexithymia and poorer quality of life across all domains as compared with healthy controls. (2) There was no significant difference in alexithymia and quality-of-life scores across diagnostic subgroups of SFD. (3) On correlation analysis, greater alexithymia scores were associated with poorer quality of life in the patient group. (4) Poorer emotion recognition ability in the patient group was associated with greater alexithymia scores. (5) Interestingly, performance on emotion recognition task was poorer in patients of somatoform pain disorder group as compared with other diagnostic subgroups. (6) On correlation analysis, the association between emotion recognition deficits and alexithymia was significant only in subgroup of patients with somatoform pain disorder.

\section{Alexithymia in SFD}

The results of this study demonstrated that patients with SFD as a group had significant alexithymia as compared with the control group. These results are consistent with findings of earlier studies which have demonstrated greater alexithymia scores in SFD. ${ }^{11-15,17,21}$ Rates of alexithymia were $62.8 \%$ in our study which was comparable to a rate of $59 \%$ reported by Burba et al. ${ }^{15}$ In the study by Bankier et al, ${ }^{29}$ factor 1 "difficulty in identifying feelings" was associated with SFD. Among the factors of TAS-26, we found TAS-4 "inability to interpret bodily manifestations of emotions" to be significantly different in the patient group as compared with control subjects which is consistent with the understanding of the concept of somatization.

\section{Alexithymia, Facial Emotion Recognition, and Its Relation to Diagnostic Subtypes}

In our study, we found that greater number of symptoms experienced was associated with a poorer quality of life 
in the physical domain and TAS-2 "externally oriented thinking" was associated with poorer quality of life in the psychological domain. An important finding of this study was that the association between alexithymia and quality of life was significant only in the diagnostic subgroup of persistent somatoform pain disorders and not in other subgroups. This finding is supported by findings of similar study which reported that the total score on TAS-20 and the factor "difficulty describing feelings" was a significant predictor of psychological domain of quality of life in patients with persistent somatoform pain disorders. ${ }^{17}$ In another study, Cox et $\mathrm{al}^{13}$ demonstrated $53 \%$ rate of alexithymia in SFD patients with chronic pain.

It is interesting to note that in our study, though there was no significant difference in alexithymia scores between the two diagnostic subtypes of SFD, the association between alexithymia and quality of life was significant only in patients with a diagnosis of persistent somatoform pain disorder. This indicates that the presence of pain symptoms is an important contributing factor determining a poor quality of life. A cognitive style of somatic attribution has been described to be associated with SFD and is seen more frequently in these patients than in depression. ${ }^{30}$ These cognitive styles possibly act as a mediator between alexithymia and expression of psychological distress as pain symptoms. This is supported by Geenen et $\mathrm{al}^{31}$ who demonstrated that better emotion expression had lower fibromyalgia impact. Schönenberg et $\mathrm{al}^{21}$ had found increased alexithymia among patients of persistent SFD. But they found no significant difference in facial emotion recognition.

We found significant difference in facial emotion recognition between patients and controls. Between diagnostic subgroups, patients with pain disorder had significantly greater deficits. There was a correlation between TAS-1 "difficulty in identifying feelings and bodily sensations" and facial emotion recognition deficits. Neurobiological markers, such as facial emotion recognition deficits have been described to be associated with concurrent alexithymia in SFD. ${ }^{21}$ Neuroanatomical substrates involved in facial emotion recognition like amygdala, insula, and anterior cingulate gyrus and orbitofrontal cortex are also found to play a part in alexithymia. ${ }^{19}$ This could be a possible explanation for facial recognition deficits found in individuals with alexithymia. It has been proposed that these deficits in emotion perception could negatively influence social functioning in these patients and hence, contribute to a poor quality of life.

We propose a model to explain interaction between alexithymia, emotion recognition deficits, and quality of life. The inability to process facial emotions possibly represents a neurobiological substrate related to alexithymia in individuals with SFD. The presence of alexithymia in individuals with a cognitive style of somatic attribution could lead to expression of psychological distress in the form of pain symptoms. Impaired emotion processing is known to be associated with impaired social functioning. This coupled with an experience of pain symptoms could lead to social reclusivity and an impaired quality of life, especially in the domain of social relationships (QOL-3).

The new DSM V classification of mental disorders removes other subtypes of somatic symptom-related disorders and retains pain as a specifier. Findings of our study underline the importance of persisting pain as a differentiating factor due to its neurological and psychological correlates.

The study findings need to be interpreted considering that $14 \%$ of patients had comorbid diagnosis of dysthymia and the patient group had significantly higher HADS score as compared with controls. Also, 15 patients had significant depressive and anxiety symptoms as indicated by score greater than 11. Patients with depression are also known to experience alexithymia and hence, presence of depressive features may confound our results. In this study, structured assessments were not used to rule out comorbid psychiatric disorders. However, all patients had been evaluated by comprehensive clinical interview and mental status examination independently by two psychiatrists and syndromal depressive and anxiety disorders had been ruled out based on ICD-10 criterion. Statistical comparisons were performed controlling for this potential confounding effect by using HADS scores as a covariate. The group of somatoform autonomic dysfunction could not be included in our analysis, as we did not have adequate number of patients of this diagnostic subtype. Internationally, the TAS-20 with three factors is used in alexithymia research since 1994 . However, the Kannada version of the 20-item scale is not available and hence, we had to use the 26-item scale in our study. All the tools used in the study were validated for Indian population. All self-reporting tools were in subjects' mother tongue, i.e., Kannada.

\section{CLINICAL IMPLICATIONS AND FUTURE DIRECTIONS}

Alexithymia is an important trait seen in up to 50 to $60 \%$ of SFD patients. This trait can adversely influence quality of life, especially in patients with a diagnosis of persistent somatoform pain disorder and hence, is of important clinical relevance. Alexithymia has been described to be a predictor of persistent somatization in 2-year longitudinal studies, indicating that presence of this trait could be poor prognostic factor. As per a recent study by da Silva et $\mathrm{al}^{32}$ emotional awareness and emotional differentiation mediate the relationship between alexithymia and 
emotion regulation. Being aware of the differential effect each alexithymia factor has on emotional processing may be helpful to guide intervention. Psychological interventions focusing on reattribution of somatic experiences and encouraging "identifying feelings" could play a greater role in the management of patients with alexithymia and hence, improving their quality of life. Also, there is a possibility of improving social cognition in order to improve symptoms and quality of life in patients of SFD, especially in patients with pain as the predominant symptom.

The findings of this study need to be replicated in a case-control design of larger sample with adequate number of patients of each diagnostic subtype. Longitudinal studies also need to be performed to validate alexithymia as a trait and its influence on long-term prognosis in SFD.

\section{CONCLUSION}

The results of our study suggest that patients of SFD have greater alexithymia and poorer quality of life as compared with healthy controls. A novel observation of clinical significance in our study was this association between alexithymia and facial emotion recognition which is predominant in patients with somatoform pain disorder. Psychological interventions focusing on "identifying feelings," and improving social cognition could potentially play a role in improving the quality of life in patients with persistent somatoform pain disorder.

\section{ACKNOWLEDGMENT}

The authors acknowledge all subjects for their participation in the study.

\section{REFERENCES}

1. Ustun, TB.; Sartorius, N. Mental illness in general health care. Chichester: Wiley; 1995.

2. Kroenke K, Swindle R. Cognitive-behavioral therapy for somatization and symptom syndromes: a critical review of controlled clinical trials. Psychother Psychosom 2000 JulAug;69(4):205-215.

3. Sharpe M, Carson A. "Unexplained" somatic symptoms, functional syndromes, and somatization: do we need a paradigm shift? Ann Intern Med 2001 May;134(9 Pt 2):926-930.

4. Ormel J, VonKorff M, Ustun TB, Pini S, Korten A, Oldehinkel T. Common mental disorders and disability across cultures: results from the WHO Collaborative Study on Psychological Problems in General Health Care. JAMA 1994 Dec;272(22):1741-1748.

5. Steckel, W.; Paul, C.; Gutheil, EA. The interpretation of dreams: new developments and technique. New York: Liveright; 1943.

6. Kellner R. Somatization: theories and research. J Nerv Ment Dis 1990 Mar;178(3):150-160.

7. Duddu V, Isaac MK, Chaturvedi SK. Alexithymia in somatoform and depressive disorders. J Psychosom Res 2003 May; 54(5):435-438.
8. Sifneos PE. The prevalence of "Alexithymic" characteristics in psychosomatic patients. Psychother Psychosom 1973;22(2):255-262.

9. Feldmanhall O, Dalgleish T, Mobbs D. Alexithymia decreases altruism in real social decisions. Cortex 2013 Mar;49(3):899-904.

10. Sriram TG, Chaturvedi SK, Gopinath PS, Subbakrishna DK. Assessment of alexithymia: psychometric properties of toronto alexithymia scale (TAS)—a preliminary report. Indian J Psychiatry 1987 Apr;29(2):133-138.

11. Sriram, TG. Alexithymia: a controlled study in patients with psychogenic pain disorder. Thesis submitted to Bangalore University, Bengaluru. 1986

12. Bach M, Bach D Alexithymia in somatoform disorder and somatic disease: a comparative study. Psychother Psychosom 1996;65(3):150-152.

13. Cox BJ, Kuch K, Parker JD, Shulman ID, Evans RJ. Alexithymia in somatoform disorder patients with chronic pain. J Psychosom Res 1994 Aug; 38(6):523-527.

14. Steinweg DL, Dallas AP, Rea WS. Fibromyalgia: unspeakable suffering, a prevalence study of alexithymia. Psychosomatics 2011 May-Jun;52(3):255-262.

15. Burba B, Oswald R, Grigaliunien V, Neverauskiene S, Jankuviene $\mathrm{O}$, Chue P. A controlled study of alexithymia in adolescent patients with persistent somatoform pain disorder. Can J Psychiatry 2006 Jun;51(7):468-471.

16. Garcia Nuñez D, Rufer M, Leenen K, Majohr KL, Grabe H, Jenewein J. Quality of life and alexithymia in somatoform pain disorder. Schmerz 2010 Feb;24(1):62-68.

17. Subric-Wrana C, Beutel ME, Knebel A, Lane RD. Theory of mind and emotional awareness deficits in patients with somatoform disorders. Psychosom Med 2010 May;72(4):404-411.

18. Larsen JK, Brand N, Bermond B, Hijman R. Cognitive and emotional characteristics of alexithymia: a review of neurobiological studies. J Psychosom Res 2003 Jun;54(6):533-541.

19. Kano M, Fukudo S. The alexithymic brain: The neural pathways linking alexithymia to physical disorders. Biopsychosoc Med 2013 Jan;7(1):1.

20. Pedrosa Gil F, Ridout N, Kessler H, Neuffer M, Schoechlin C, Traue HC, Nickel M. Facial emotion recognition and alexithymia in adults with somatoform disorders. Depress Anxiety 2009;26(1):E26-E33.

21. Schönenberg M, Mares L, Smolka R, Jusyte A, Zipfel S, Hautzinger M. Facial affect perception and mentalizing abilities in female patients with persistent somatoform pain disorder. Eur J Pain 2014 Aug;18(7):949-956.

22. Shamasunder C, Sriram TG, Murali Raj SG, Shanmugham V. Validity of a short 5-item version of the general health questionnaire (g.h.q). Indian J Psychiatry 1986 Jul-Sep;28(3):217-219.

23. Taylor GJ, Ryan DP, Bagby RM. Towards the development of a new self-report alexithymia scale. Psychother Psychosom 1985;44(4):191-199.

24. Wise TN, Simpson N, Sheridan MJ. Comparison of 26-item and 20-item versions of the Toronto Alexithymia Scale for psychiatric outpatients. Psychol Rep 2000 Aug;87(1):127-132.

25. Tacchini, G.; Janca, A.; Isaac, M. Somatoform disorder schedule who international study of somatoform disorders study protocols and instruments. Geneva: WHO; 1993.

26. Skevington SM, Lotfy M, O'Connell KA; WHOQOL Group. The World Health Organization's WHOQOL-BREF quality of life assessment: Psychometric properties and results of the international field trial. A Report from the WHOQOL Group. Qual Life Res 2004 Mar;13(2):299-310. 
27. Snaith RP. The hospital anxiety and depression scale. Health Qual Life Outcomes 2003 Aug;1:29.

28. Behere RV, Raghunandan V, Venkatasubramanian G, Subbakrishna DK, Jayakumar PN, Gangadhar B. TRENDS- A tool for recognition of emotions in neuropsychiatric disorders. Indian J Psychol Med 2008;30(1):32-38.

29. Bankier B, Aigner M, Bach M. Alexithymia in DSM-IV disorder: comparative evaluation of somatoform disorder, panic disorder, obsessive-compulsive disorder, and depression. Psychosomatics 2001 May-Jun;42(3):235-240.
30. Duddu V, Isaac MK, Chaturvedi SK. Somatization, somatosensory amplification, attribution styles and illness behaviour: a review. Int Rev Psychiatry 2006 Feb;18(1):25-33.

31. Geenen R, van Ooijen-van der Linden L, Lumley MA, Bijlsma JW, van Middendorp H. The match mismatch model of emotional regulation strategies in fibromyalgia. J Psychosom Res 2012 Jan;72(1):45-50.

32. da Silva AN, Vasco AB, n JC. Alexithymia and emotional processing: a mediation model. J Clin Psychol 2016 Dec;73(9): 1196-1205. 